\title{
EDITORIAL
}

\section{CLINICAL AND LABORATORY ASSESSMENT OF NUTRITIONAL STATUS}

\author{
Mário Cícero Falcão*
}

Pediatricians, experts in treating patients as a whole, are in a perfect position to diagnose nutritional disease or deficiency. To make a correct diagnosis, we must first be aware of the symptoms of nutrients and energy deficiencies and/or excesses. Nutritional injuries usually do not announce their presence, they must be sought.

There is consensus in the scientific community that a relationship between diet and health exists. As in other areas of clinical medicine, a careful medical, socioeconomic, and dietary history must be obtained.

Because pediatricians have the capability of assessing growth rates in their patients, they should emphasize the utilization of these data in providing information about nutritional status.

Anthropometric measurements, in relation to nutritional status, can be thought of predominantly as methods of assessment of protein-calorie adequation. Growth curves for an individual, utilizing measurements of weight, length, and head circumference, offer one of the best guides to the nutritional status of the child. Measurements of skinfold thickness and arm circumference can provide estimates of body composition that are useful for interpretation along the length, weight, and head circumference data. Anthropometric measurements are considered precise data and should be performed carefully by well-trained and well-supervised individuals using appropriate instruments.

The assessment of nutritional status by laboratory tests potentially offers a reproducible quantitative means of measuring specific nutrients that can be of great use to clinicians, nutritionists, and researches. It can provide objective confirmation of nutritional deficiencies. Laboratory tests can also be used to monitor nutritional therapy with greater precision compared to separate use of dietary, anthropometric, or clinical assessment techniques. They may be used to determine quantitative alterations in biochemical levels of nutrients, their metabolites, or dependent enzyme activities that are often not detected by anthropometric methods.

However, despite the availability of a myriad of biochemical and immunologic assays, the laboratory assessment of nutritional status has so far failed to fulfill its promise. There have been no widely accepted methods to integrate information about the various nutrients that need to be screened into a battery of a few standard, readily available and inexpensive tests. Part of the reason for this situation may be that most laboratory tests of nutritional status are too specific. Although they nicely quantitate levels of a certain nutrient in a specific 
body fluid at a particular time, these measurements may not correlate with values at other times, in other body pools, or with deficiencies of other nutrients. Furthermore, many drugs, diseases, and end environmental con- ditions not related to nutrition can affect measured levels of nutrients.

In summary, unfortunately, no test gives a completely satisfactory picture of the global nutritional state of the child. Thus, laboratory assessment of nutri- tional status requires an intelligent selection of tests to fit the clinical situation. It should augment the dietary, clinical, and anthropometric data that should also be gathered and evaluated and should not be excluded from nutritional assessment. 